\title{
Guidelines of the Italian Society of Videosurgery in Infancy (SIVI) for the minimally invasive treatment of fetal and neonatal ovarian cysts
}

\author{
Salvatore Fabio Chiarenza,1 Maria Luisa Conighi,1 ${ }^{1}$ Andrea Conforti,2 Cosimo Bleve, ${ }^{1}$ Ciro Esposito, ${ }^{3}$ \\ Maria Escolino, ${ }^{3}$ Fabio Beretta, ${ }^{4}$ Maurizio Cheli,5 Vincenzo Di Benedetto, ${ }^{6}$ Maria Grazia Scuderi, 6 \\ Giovanni Casadio, ${ }^{7}$ Maurizio Marzaro, ${ }^{8}$ Marco Gambino, ${ }^{9}$ Alessio Pini Prato, ${ }^{10}$ Francesco Molinaro, ${ }^{11}$ \\ Simona Gerocarni Nappo, ${ }^{12}$ Paolo Caione13 \\ 1Pediatric Surgery and Urologic Unit, Regional Center of Minimally Invasive Surgery and Urology, S. Bortolo Hospital, \\ Vicenza; ${ }^{2}$ Congenital Esophageal Disorders Unit, Neonatal Surgery Unit, Bambino Gesù Children's Research Hospital, \\ Rome; ${ }^{3}$ Pediatric Surgery Unit, Federico II University, Naples; ${ }^{4}$ Pediatric Surgery Unit, APSS, Trento; 5 Pediatric Surgery \\ Department Papa Giovanni XXIII Hospital, Bergamo; ${ }^{6}$ Pediatric Surgery Unit, Vittorio Emanuele Hospital, Catania; \\ ${ }^{7}$ Pediatric Surgery Unit, Parma University Hospital, Parma; ${ }^{8}$ Pediatric Surgery Unit, Local Health Unit 2, Treviso Hospital, \\ Treviso; 'Pediatric Surgery Unit, Annunziata Civil Hospital, Cosenza; ${ }^{10}$ Unit of Pediatric Surgery, The Children Hospital, \\ Azienda Ospedaliera SS Antonio e Biagio e Cesare Arrigo, Alessandria; ${ }^{11}$ Department of Medical Sciences, Surgery and \\ Neuroscience, Section of Pediatric Surgery, University of Siena, Siena; 12 Pediatric Surgery Unit, Regina Margherita \\ Hospital, Turin; ${ }^{13}$ Pediatric Surgery and Urologic Unit, Pediatric Hospital Bambino Gesù, Rome, Italy
}

\section{Background}

The SIVI (Italian Society of Videosurgery in Infancy) guidelines are clinical practice guidelines edited and approved by the Society's steering committee. They are the products of a detailed systematic review of the literature, integrated with expert opinion in the field of pediatric minimally invasive surgery. These guidelines are intended to assist the pediatric surgeons experienced or not experienced in minimally invasive surgery, with the goal to inform choices related to the indications, approach, and techniques to use when treating the major pediatric surgical pathologies.

Given the complexities of congenital malformations and other pediatric surgical conditions, as well as large variations in available regional health services, we must note that these guidelines are not intended as a cookbook recipe to follow for all possible patients. Rather, the guidelines should serve as a flexible framework, to be

Correspondence: Salvatore Fabio Chiarenza, Pediatric Surgery and Urologic Unit, Regional Center of Minimally Invasive Surgery and Urology, S. Bortolo Hospital, viale Rodolfi 37, 36100, Vicenza, Italy. Tel.: +39.0444.752642.

Fax: +39.0444 .752643$

E-mail: fabio.chiarenza@aulss8.veneto.it

Key words: Fetal Ovarian Cyst; Fetal Ovarian Torsion; Prenatal Diagnosis; Ultrasound; Laparoscopy.

Received for publication: 8 October 2020.

Accepted for publication: 8 October 2020.

This work is licensed under a Creative Commons Attribution

NonCommercial 4.0 License (CC BY-NC 4.0).

OCopyright: the Author(s), 2020

Licensee PAGEPress, Italy

La Pediatria Medica e Chirurgica 2020; $42: 242$

doi:10.4081/pmc.2020.242 used by the physician in concert with the parents, to choose the best approach for each individual patient. Decisions tailored to available scientific knowledge and the needs and desires of the patient's family serve both patient autonomy and medical science.

All guidelines are published in this scientific Journal, in order to ensure their availability to all physicians.

The Guidelines project has been approved by the SIVI General Assembly of the 2016 Madrid congress.

Review of guidelines has been performed by the Steering Committee of SIVI and experts.

\section{Introduction}

In the last three decades, fetal ovarian cysts were diagnosed more frequently, due to technological improvement and the increasing use of prenatal screening ultrasound. Nonetheless, treatment uncertainties are still present, either prenatally or postnatally. Recently, significant innovations on diagnosis and treatment have been proposed and a more conservative, minimally invasive approach may be offered to the Pediatrician or the Surgeon who face with this condition during prenatal or neonatal age.

These recommendations have been approved by the SIVI Board of Directors and are developed as a flexible guide to correctly address the Gynecologist, Pediatric Surgeon or Urologist and also the Neonatologist to choose the most appropriate treatment, selected from the updated literature and from the experience of the SIVI group of Experts.

\section{Definition and epidemiology}

The exact incidence of Fetal and Neonatal Ovarian Cysts (FNOC) is not known, usually reported as high as 1 in 1000-2500 live born. ${ }^{1,2}$ During fetal and neonatal life, ovaries are usually considered quiescent, and no consensus on the development of FNOC 
exists. The wider accepted theory implicates an abnormal response to maternal gonadotropin and estrogen, thought to induce follicular cysts growth and their pseudo-maturation.2,3 Enriquez and co-workers suggest a possible role of gonadic dysgenesis, secondary to vascular injury, as risk factor for development of complicated cysts. ${ }^{4}$

Generally discovered during 3rd trimester, FNOC are usually not considered pathologic if less than $20 \mathrm{~mm}$ in diameter, while is common the ultrasonographic incidental finding of stimulated ovaries, with cysts ranging 1 or $2 \mathrm{~mm}$ in diameter.

Ovarian malignant development during fetal and neonatal life is extraordinary rare, with a single case reported in international literature: 5 an analysis of 91 cases of pediatric ovarian malignancies did not demonstrated any tumor development before the $1^{\text {st }}$ year of life. ${ }^{7}$

\section{Prenatal diagnosis}

A sonographic-pathologic correlation and FNOC classification was firstly proposed by a multidisciplinary group from Johns Hopkins Medical Institution in 1988.8,9 FNOC may present as SIMPLE or COMPLEX lesions.

Ultrasonographic appearance of SIMPLE FNOC include round, anechoic, single cyst with thin wall, usually unilateral with pelvic position. 3,8 Occasionally, simple FNOC may present an internal septum, while the presence of a daughter cyst (a smaller cyst within the FNOC) is recognized as pathognomonic diagnostic sign:3,8,9 no other abdominal cystic lesions (including lymphangiomas, intestinal duplication, meconium pseudo-cyst, hydrometrocolpos or uracal cyst) were ever been reported with daughter cyst evidence $^{9}$ (Figure 1).

At the ultrasonographic scan, COMPLEX FNOC are characterized by a thickened wall with heterogenic appearance: multiple septa are commonly reported, associated with debris flocculation and/or fluid-fluid level, or solid feature. ${ }^{3}$ It is often thought that complex FNOC are the result of ovarian torsion, occurring more frequently during fetal life more than neonatal period. ${ }^{10}$ In some cases, complex FNOC are associated to poly-hydramnios and/or fetal ascites, interpreted as cyst rupture or cyst exudation. ${ }^{11,12}$ Moreover, intestinal occlusions were reported secondary to necrosis of complicated FNOC developing intestinal adherences. ${ }^{10}$ Therefore, a diagnosis of complicated FNOC is commonly considered as an indication for surgical postnatal exploration 3,10 (Figure 2).

\section{Prenatal MRI}

In selected cases, specifically when the ultrasonographic window is limited by maternal habitus, fetal position or important polyhydramnios, fetal MRI could be helpful. Furthermore, MRI could also be useful to confirm the presence of female genitalia, gastrointestinal and genitourinary anatomy, which are necessary in the diagnosis of fetal ovarian cysts and in their differential diagnosis. 3,13

\section{Differential diagnosis}

Differential diagnosis of intra-abdominal fetal cysts is broad. Nonetheless, when is possible to establish the organ of origin, it is also possible to quickly limit the differential diagnostic entities, decisively orienting prenatal counseling, pre and postnatal treat- ment. ${ }^{3}$ The main cystic lesions that must enter into differential diagnosis with fetal ovarian cysts are: simple renal cysts, multicystic dysplastic kidney, hydronephrosis, ureterocele, uracal cysts, hydrocolpos, enteric cystic duplications, meconial pseudocysts, lymphangiomas, fetus in fetu. Each of these entities presented specific ultrasonographic and MRI characteristics (both pre-and postnatally) that usually allow a correct diagnosis.

\section{Clinical evolution}

Following the prenatal diagnosis of ovarian cyst, an ultrasound (US) follow up is indicated until delivery to monitor any changes in the size and/or echogenicity of the cyst. US timing is established according to the diameter and the characteristics of the lesion.

As reported in literature, the size of the cyst correlate both with the risk of fetal ovarian torsion and with the rate of spontaneous resolution. Even if there is no clear consensus on a defined diame-

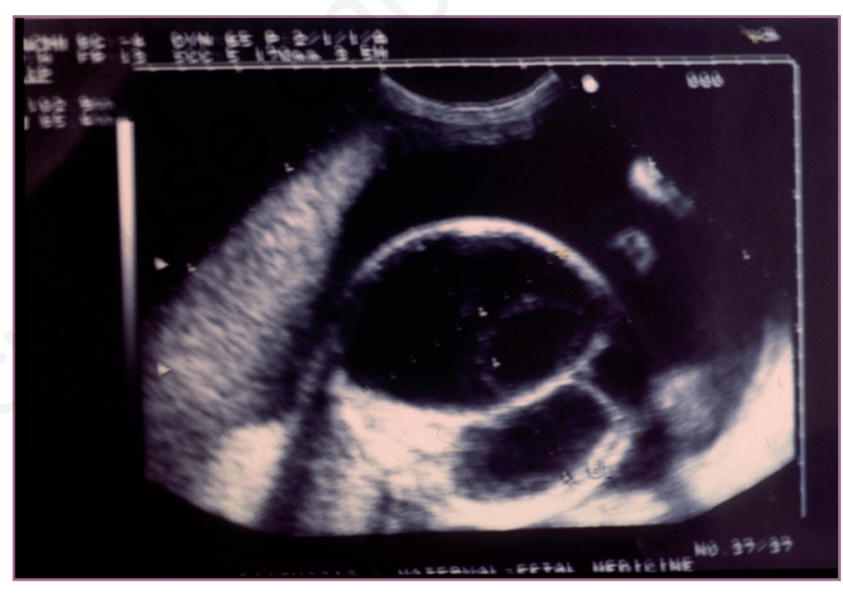

Figure 1. Simple FNOC with daughter cyst.

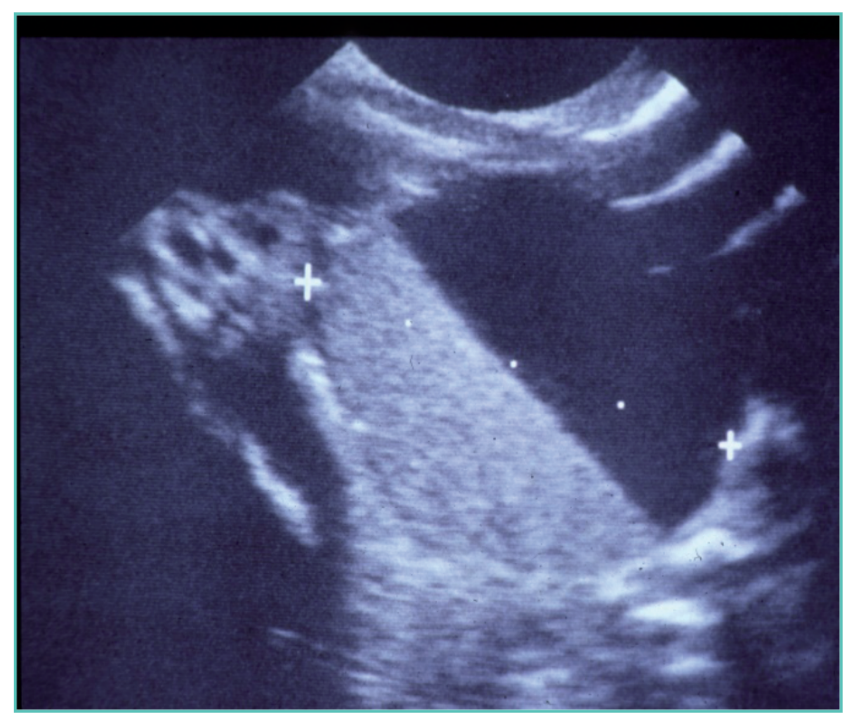

Figure 2. Complex FNOC. 
ter (in different studies diameters from 3 to $6 \mathrm{~cm}$ are considered as cut-off), it can be assumed that simple cysts $\leq 4 \mathrm{~cm}$ will undergo more frequently complete regression during prenatal or postnatal period; while simple cysts $>4 \mathrm{~cm}$ more frequently are associated with torsion or with postnatal surgery. $3,10,14,15$ Moreover, most complications of fetal ovarian cysts (intracystic hemorrhage, necrosis, adhesions, compression of the surrounding organs, infertility, etc.) are due to the torsion itself. Because of that, simple cysts $>4 \mathrm{~cm}$, or with a growth of more than $1 \mathrm{~cm}$ per week, have to be monitored very closely and fetal aspiration could be considered. ${ }^{3,10}$

Changes in US pattern of the cyst during follow up are associated with an increased risk of ovarian loss (autoamputation or postnatal surgical removal). 16

Complicated cysts have a significant lower rate of spontaneous resolution compared to simple cysts. Nonetheless, they could not be considered an indication for preterm labor: the torsion event usually has an undefined duration and the loss of ipsilateral ovarian function cannot be correlated to the time of delivery.

Considering simple ovarian cysts, conservative postnatal management (clinical and US follow up) is suggested since most of them may undergo spontaneous regression. Postnatal US scans can be scheduled every 3-6 weeks according to the diameter and the characteristics of the lesion. US follow up is continued until cyst regression or until the appearance of complications (size increase, torsion) or, if the lesion persists, until the age of 6 month. 12

The risk of having postnatal surgery is higher in cysts $>4 \mathrm{~cm}$ compared with those $\leq 4 \mathrm{~cm}$ and in complex cysts compared to simple ones (irrespective of the cyst size). 16

If an increase in cyst size is observed, clinical symptoms develop or the cyst persist beyond 6 months, an operative approach is indicated to reach a correct diagnosis. ${ }^{17}$

Operative treatment of ovarian cyst could offers several options: non-surgical (aspiration reserved to simple ovarian cysts) or surgical (derotation, deroofing, cystectomy, oophorectomy, salpingo-oophorectomy). Considering the single hospital attitude and experience, surgery can be performed with a minimally invasive approach or with a traditional open approach. Laparoscopic surgery has proved to be feasible and safe in cases of neonatal ovarian cysts, and in centers of expertise, should be considered as first approach. ${ }^{12,18}$

\section{Treatment}

\section{Prenatal treatment (Figure 3)}

The risk of fetal ovarian torsion has led several groups to perform ultrasound-guided prenatal aspiration in cases of large simple ovarian cysts. ${ }^{4}$

However, it is mandatory to balance the benefits of this maneuver with the potential risks of complications as bleeding and uterine contractions that can lead to preterm delivery.

To date, there is a low level of evidence in literature about the efficacy and safety of prenatal ovarian cysts aspiration. Different authors reported that simple cysts with a diameter larger than 3 or 4 or $5 \mathrm{~cm}$ (depending on author) had lower rates of torsion when aspirated prenatally and lower rates of postnatal surgery (in particular postnatal oophorectomy) compared to same size cysts conservatively managed. $3,15,19-22$

In line with this, several groups have proposed to reserve this invasive procedure only to simple ovarian cysts $>4 \mathrm{~cm} .10,23,24$

Waiting for a wider consensus, we want to highlight the need to provide mothers with excellent information and communications from physician responsible of the procedure.

\section{Postnatal treatment (Figure 4)}

It is thought that excessive maternal hormonal stimulation determines the development of the majority of fetal ovarian cysts, ${ }^{21,25}$ and that the physiological discontinuation of this hormonal stimulation after birth determines the spontaneous resolution of the cysts themselves.

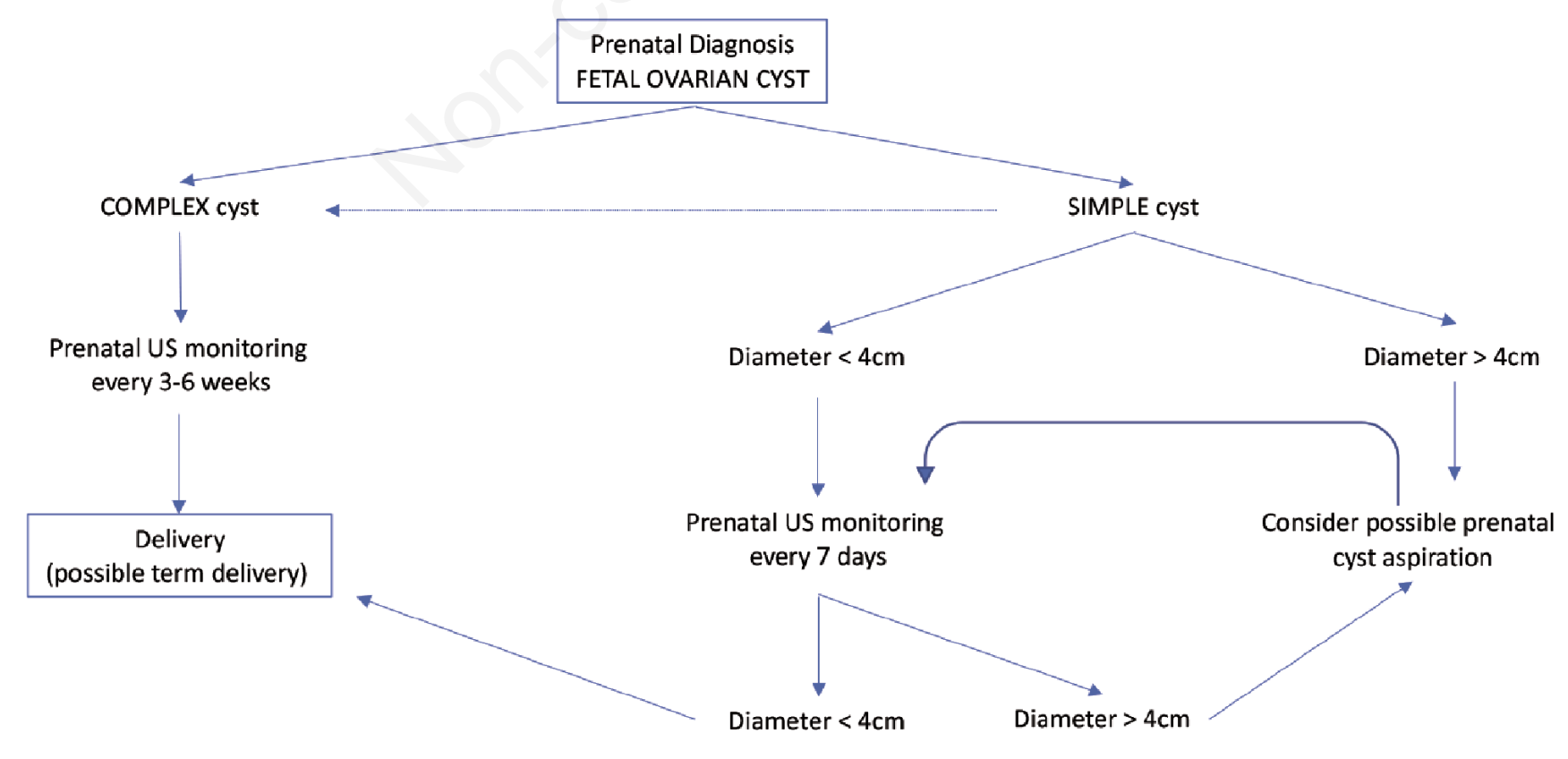

Figure 3. Flowchart: management of fetal ovarian cyst. 
In the case of simple ovarian cysts, conservative postnatal management consists in a clinical and US follow up since the cysts may spontaneously regress. Postnatal ultrasound scans can be scheduled every 3-6 weeks until cyst regression.

We can resume indications to post-natal treatment to: i) Complex cysts (diagnostic definition); ii) Symptomatic cysts (suspected torsion); iii) Simple cysts complicating their aspect during postnatal US follow up (suspected torsion); iv) Simple cysts growing more than $4 \mathrm{~cm}$ during postnatal US follow up (improved risk of torsion); v) Simple cysts persisting over the age of 6 months (diagnostic definition).

As said before, there is no clear consensus on diameter cut off.

Postnatal treatment can offer different options.

\section{US-guided cyst aspiration}

Postnatal US-guided cyst aspiration is less controversial and debated than intrauterine aspiration procedure. 26,27 It aims to reduce the risk of torsion when large simple cyst (at least $4 \mathrm{~cm}$ in maximum diameter) is confirmed at postnatal US. ${ }^{2}$

There is few literature about this procedure: some authors proposed US-guided percutaneous aspiration as the treatment of choice for simple neonatal ovarian cysts, limiting surgical exploration as much as possible; 27 others questioned the efficacy of aspiration during neonatal period since there are no extensive studies on the subject; 21 some studies have shown the need for a second puncture to obtain a complete evacuation of the cyst, therefore even in cases of postnatal aspiration an ultrasound follow-up period is usually indicated. 28

In conclusion, advantages of this procedure could be considered effectiveness and repeatability, moreover some authors suggest that this procedure preserve ovarian tissue in a higher percentage of patient than surgery. ${ }^{26-28}$ Nonetheless some reports question US diagnostic accuracy and the risk to puncture different lesions than ovarian cysts (e.g. enteric duplication). ${ }^{29}$

\section{Surgical approach}

Some authors have shown a close correlation between the diameter of the cysts $(>5 \mathrm{~cm})$ and the need for surgical exploration: 12 the laparoscopic approach has proved to be safe and effective in numerous works. ${ }^{12,18}$

The aim of surgery has to be the more conservative as possible on ovarian tissue: fenestration of the cyst, deroofing and cystectomy should be considered in cases of simple ovarian cyst in order to preserve the ovarian parenchyma. ${ }^{30,31}$

If ovarian torsion occurs during post-natal follow-up, a surgical attempt at ovarian de-torsion must be undertaken to spare the ovary even if the pedicle is usually very thin. Oophorectomy and/or salpingo-oophorectomy should be reserved to those cases of irreversible ovarian necrosis or in which torsion places the patient at risk of sepsis, or hemodynamic instability.

Usually, the presence of complex ovarian cysts indicates the need for surgical exploration: some authors proposes surgical excision in case of neoplastic suspicion, in cases of complex ovarian cysts and when simple cysts remain unchanged for more than 6 months. ${ }^{10,32,33}$ However, the finding of complex ovarian cysts does not place an absolute indication to surgery: ${ }^{3,34}$ authors reported a spontaneous tendency towards involution of twisted ovarian cysts, concluding that conservative treatment of such cysts may represent a therapeutic option and that surgery should be reserved for particularly large lesions (when it results in an intra-abdominal mass effect) or those that do not tend to involve. ${ }^{4}$

However, conservative treatment of complex ovarian cysts, however, does not reduce the risks of possible complications, which, although rare, have important clinical implications: ${ }^{21}$ there are several publications reporting the correlation between intracystic hemorrhage and development of fetal, or neonatal, anemia requiring transfusion therapy; 35,36 moreover postnatal intestinal obstruction caused by complex ovarian cysts is also described: the presence of self-amputated ovarian cysts can cause inflammation

\section{COMPLEX ovarian cyst}

\section{SIMPLE ovarian cyst}

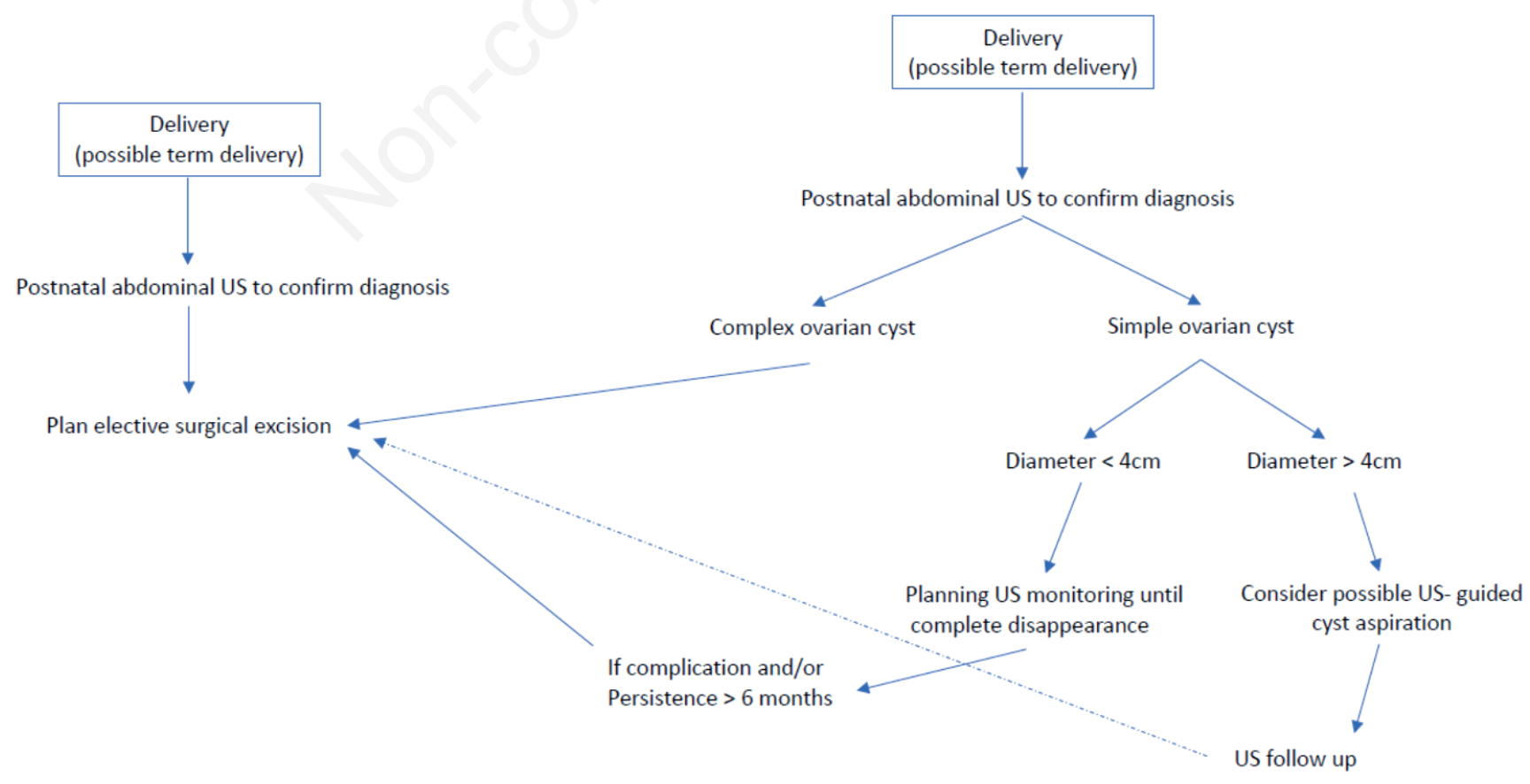

Figure 4. Flowchart: postnatal management of ovarian cyst. 
and intestinal adhesions (the risk of intestinal obstruction due to amputated cysts has not been compared to that caused by laparoscopic exploration, and there are divergent opinions on this aspect). $37-39$

When surgery is needed, different minimally invasive techniques are described to approach neonatal ovarian cyst: open (transumbilical approach); videoassisted technique (one trocar); laparoscopic (three trocars). ${ }^{40-44}$

As described in literature, all these minimally invasive approaches have to be considered diagnostic and therapeutic. They are safe and effective, operation time and length of hospital stay are short and cosmetic appearance after surgery is good.

It is important to remind that intraperitoneal spillage of cystic liquid could happen during these procedures, so it is important to exclude a neoplastic lesion before surgery (even if they are extremely rare in neonatal period).

Before the operation, a nasogastric tube is positioned and the cleaning of the bowel with one or more enema is recommended:

i) Transumbilical technique: because of the long ovarian pedicle, which consent a good mobility, and the frequent intrabdominal position of the neonatal ovarian cyst, it is possible to manage the lesion through a transumbilical route. A semicircular infraumbilical incision (according to Bianchi) is made, the fascia is longitudinally incised and the abdomen is entered. The cystic surface is then exposed through the incision and the liquid content is aspired by a needle (it is recommended to send a sample to cytological analysis). The partially collapsed cyst is then exteriorized for derotation/deroofing/cystectomy/oophorectomy/salpingooophorectomy.

ii) Video-assisted technique: through a trans or infra-umbilical incision a 5 or $10 \mathrm{~mm} 30^{\circ}$ laparoscope with operative channel is inserted in the abdominal cavity and pneumoperitoneum is induced up to $6 \mathrm{mmHg}$. Ovarian cyst is then visualized and have to be aspirated. Aspiration could be performed with different techniques: thanks to the operative trocar the cyst could be mobilized and expose through the umbilical incision to proceed aspiration and surgery as described for the transumbilical approach. Alternatively, the ovarian cyst is approached to the abdominal wall thanks to a forceps, and, under laparoscopic control, a needle is inserted through the abdominal wall to aspire the cyst; the cyst partially collapsed is then exteriorized through the umbilical incision and procedure is completed as described before.

iii) Laparoscopic technique: a 3 or $5 \mathrm{~mm} 30^{\circ}$ laparoscope is inserted through a transumbilical incision; pneumoperitoneum induced up to $6 \mathrm{mmHg}$; two $3 \mathrm{~mm}$ operative trocars are positioned under direct vision in right and left hypochondriac regions ( according the triangulation and the position of the cyst that could be not necessarily in the pelvis ).If a torsion is diagnosed, the ovary is de-rotate thanks to the operative instruments. To aspire the cyst is possible to incise its surface with a Crochet hook needle and insert through this incision an endoscopic aspirator to empty the cyst. Then is possible to proceed laparoscopically with deroofing/cystectomy/oophorectomy/salpingo.oophorectomy.

\section{Conclusions}

As final recommendations, the evidence currently available on the diagnosis and treatment of fetal ovarian cysts observed in prenatal and postnatal life is very limited, since no randomized studies are available on any aspect of this entity. Often the indications for treatment derive from revisions of small monocentric case studies. Nevertheless, we can conclude that prenatal treatment with ultrasound-guided puncture of simple ovarian cysts $>40 \mathrm{~mm}$ seems to be related to a significant benefit in terms of preservation of the ovarian parenchyma. Postnatal surgical approach in cases complicated by ovarian torsion appears to be useful to safeguarding the residual ovarian parenchyma and preventing possible complications secondary to ovarian torsion, as shown in the flowchart.

\section{References}

1. Valenti C, Kassner EG, Yermakov C, et al. Antenatal diagnosis of a fetal ovarian cyst. Am J Obstet Gynecol 1975;123: 216-21.

2. Bryant AE, Laufer MR. Fetal ovarian cysts: incidence, diagnosis and management. J Reprod Med 2004;49:329-37.

3. Trinh TW, Kennedy AM. Fetal Ovarian Cysts: Review of Imaging Spectrum, Differential Diagnosis, Management, and Outcome. Radiographics 2015;35:621-35.

4. Enríquez G, Durán C, Torán N, et al. Conservative versus surgical treatment for complex neonatal ovarian cysts: outcomes study. AJR Am J Roentgenol 2005;185: 501-8.

5. Heaton TE, Liechty KW. Postnatal management of prenatally diagnosed abdominal masses and anomalies. Prenat Diagn 2008; 28: 656-66.

6. Heling KS, Chaoui R, Kirchmair F, et al. Fetal ovarian cysts: prenatal diagnosis, management and postnatal outcome. Ultrasound Obst Gyn 2002;20:47-50.

7. Brown MF, Hebra A, McGeehin K, et al. Ovarian masses in children: a review of 91 cases of malignant and benign masses. J Pediatr Surg 1993;28:930-3.

8. Nussbaum AR, Sanders RC, Hartman DS, et al. Neonatal ovarian cysts: sonographic-pathologic correlation. Radiol 1988; 168:817-21.

9. Quarello E, Gorincour G, Merrot T, et al. The "daughter cyst sign": a sonographic clue to the diagnosis of fetal ovarian cyst. Ultrasound Obstet Gynecol 2003;22:433-4.

10. Lee HJ, Woo SK, Kim JS, Suh SJ. "Daughter cyst" sign: a sonographic nding of ovarian cyst in neonates, infants, and young children. AJR Am J Roentgenol 2000;174:1013-5.

11. Bagolan P, Giorlandino C, Nahom A, et al. The management of fetal ovarian cysts. J Pediatr Surg 2002;37:25-30.

12. Shimada T, Miura K, Gotoh H, et al. Management of prenatal ovarian cysts. Early Hum Dev 2008;84:417-20.

13. Zampieri N, Borruto F, Zamboni C, Camoglio FS. Foetal and neonatal ovarian cysts: a 5-year experience. Arch Gynecol Obstet 2008;277:303-6.

14. Tyraskis A, Bakalis S, Scala C, et al. A retrospective multicenter study of the natural history of fetal ovarian cysts. J Pediatr Surg 2018;53:2019-22.

15. Tyraskis A, Bakalis S, David AL, et al. A systematic review and meta-analysis on fetal ovarian cysts: impact of size, appearance and prenatal aspiration. Prenat Diagn 2017;37:951-8.

16. Bascietto F, Liberati M, Marrone L, et al. Outcome of fetal ovarian cysts diagnosed on prenatal ultrasound examination: systematic review and meta-analysis. Ultrasound Obstet Gynecol 2017;50:20-31.

17. Martin C, Darnell A, Escofet C, et al. Fetal MR in the evaluation of pulmonary and digestive system pathology. Insights Imaging 2012;3:277-93.

18. Chiaramonte C, Piscopo A, Cataliotti F. Ovarian cysts in newborns. Pediatr Surg Int 2001;17:171-4.

19. Diguisto C, Winer N, Benoist G, et al. In-utero aspiration vs 
expectant management of anechoic fetal ovarian cysts: open randomized controlled trial. Ultrasound Obstet Gynecol 2018;52:159-64.

20. Crombleholme TM, Craigo SD, Garmel S, et al. Fetal ovarian cyst decompression to prevent torsion. J Pediatr Surg 1997;32:1447-9.

21. Giorlandino C, Rivosecchi M, Bilancioni E, et al. Successful intrauterine therapy of a large fetal ovarian cyst. Prenat Diagn 1990;10:473-5.

22. Noia G, Riccardi M, Visconti D, et al. Invasive fetal therapies: approach and results in treating fetal ovarian cysts. J Matern Fetal Neonatal Med 2012;25:299-303.

23. Pryse-Davis J, Dewhurst CJ. The development of the ovary and uterus in the foetus, newborn and infant: a morphological and enzyme histochemical study. J Pathol 1971;103:5-25.

24. de Sa DJ. Follicular ovarian cysts in stillbirths and neonates. Arch Dis Child 1975;50:45-50.

25. Sakala EP, Leon ZA, Rouse GA. Management of antenatally diagnosed fetal ovarian cysts. Obstet Gynecol Surv 1991;46: 407-14.

26. Kessler A, Nagar H, Graif M, et al. Percutaneous drainage as the treatment of choice for neonatal ovarian cysts. Pediatr Radiol 2006;36:954-8.

27. Puligandla PS, Laberge JM. Lethal outcome after percutaneous aspiration of a presumed ovarian cyst in a neonate. Semin Pediatr Surg 2009;18:119-21.

28. Mizuno M, Kato T, Hebiguchi T, et al. Surgical indications for neonatal ovarian cysts. Tohoku J Exp Med 1998;186:27-32.

29. Galinier P, Carfagna L, Juricic M, et al. Fetal ovarian cysts management and ovarian prognosis: a report of 82 cases. J Pediatr Surg 2008;43:2004-9.

30. Shimada T, Miura K, Gotoh H, et al. Management of prenatal ovarian cysts. Early Human Development 2008;84:417-20.

31. Luzzatto C, Midrio P, Toffolutti T, Suma V. Neonatal ovarian cysts: management and follow-up. Pediatr Surg Int 2000;16: 56-9.

32. Bagolan P, Rivosecchi M, Giorlandino C, et al. Prenatal diagnosis and clinical outcome of ovarian cysts. J Pediatr Surg 1992;27:879-81.
33. Vitezica I, Czernik C, Rothe K, et al. Prenatal diagnosis and management of a massive fetal ovarian hemorrhagic cyst torsion with secondary fetal anemia. J Clin Ultrasound 2014;42: 219-22.

34. Abolmakarem H, Tharmaratnum S, Thilaganathan B. Fetal anemia as a consequence of hemorrhage into an ovarian cyst. Ultrasound Obstet Gynecol 2001;17:527-8.

35. Jeanty C, Frayer EA, Page R, et al. Neonatal ovarian torsion complicated by intestinal obstruction and perforation, and review of the literature. J Pediatr Surg 2010;45:e5-e9.

36. Focseneanu MA, Omurtag K, Ratts VS, et al. The auto-amputated adnexa: a review of findings in a pediatric population. $\mathrm{J}$ Pediatr Adolesc Gynecol 2013;26:305-13.

37. Lin JY, Lee ZF, Chang YT. Transumbilical management for neonatal ovarian cysts. J Pediatr Surg 2007;42:2136-9.

38. Ferro F, Iacobelli BD, Zaccara A, et al. Exteriorization-aspiration minilaparotomy for treatment of neonatal ovarian cysts. J Pediatr Adolesc Gynecol 2002;15:205-7.

39. Schenkman L, Weiner TM, Phillips JD. Evolution of the surgical management of neonatal ovarian cysts: laparoscopic-assisted transumbilical extracorporeal ovarian cystectomy (LATEC). J Laparoendosc Adv Surg Tech A 2008;18:635-40.

40. Cho MJ, Kim DY, Kim SC. Ovarian Cyst Aspiration in the Neonate: Minimally Invasive Surgery. J Pediatr Adolesc Gynecol 2015;28:348-53. doi: 10.1016/j.jpag.2014.10.003.

41. van der Zee DC, van Seumeren IG, Bax KM, Rövekamp MH, ter Gunne AJ. Laparoscopic approach to surgical management of ovarian cysts in the newborn. J Pediatr Surg 1995;30: 42-3.

42. Esposito C, Garipoli V, Di Matteo G, De Pasquale M. Laparoscopic management of ovarian cysts in newborns. Surg Endosc 1998;12:1152-4.

43. Templeman CL, Reynolds AM, Hertweck SP, Nagaraj HS. Laparoscopic management of neonatal ovarian cysts. J Am Assoc Gynecol Laparosc 2000;7:401-4.

44. Tseng D, Curran TJ, Silen ML. Minimally invasive management of the prenatally torsed ovarian cyst. J Pediatr Surg 2002;37:1467-9. 